\title{
Excitation of Polar Motion
}

\author{
Clark R. Wilson \\ Department of Geological Sciences Center for Space Research and \\ Institute for Geophysics University of Texas Austin, Austin TX 78712 \\ $U S A$
}

Abstract. Conceptual models of polar motion have evolved over the past century, as improved data revealed signals over progressively wider frequency bands. In the $1890 \mathrm{~s}$, Chandler represented polar motion as a sum of discrete components, 14 month and annual terms, and this component model effectively summarized the observations, but did not provide a physical explanation for them. Over time both the search for a physical understanding of polar motion, and the ability to observe the broad band continuum outside the Chandler and annual bands have led to an understanding of polar motion as a continuum of variations, not adequately described by a few discrete components. The continuum concept is now the working model in most studies of polar motion. The transition from component to continuum conceptual models preceded the arrival of high quality data by several decades, and reflected significant contributions from Harold Jeffreys. With modern space geodetic observations and good global numerical models of the atmosphere, oceans, and other climate processes, it is clear that air and water motion and redistribution are the dominant contributors to the excitation continuum.

\section{Conceptual Models of Polar Motion}

Evolution of physical and conceptual models of polar motion is of historical interest, but also continues to be important scientifically, because conceptual models influence questions that are posed, methods of data analysis, and ultimately scientific conclusions about the Earth. A discussion of polar motion excitation thus correctly begins with identification of the conceptual or working model that underlies the study. I identify two distinctly different models that are in current use. One, called the continuum model, treats polar motion as a linear filtered version of the excitation function. The other, called the component model, separates polar motion into discrete components of different frequencies. These two models are not exclusive of one another, but they still may lead to different conclusions, as discussed below.

It is widely understood that polar motion results when an excitation process, a continuum of time varying torques, act upon the Earth. This continuum model separates observed polar motion into two elements, first, the excitation process (which may be the sum of several effects) and second, the Earth's response, dominated by the resonance at the Chandler frequency. Under the continuum 
model, observed polar motion is a linear filtered version of the excitation process. The two elements, excitation and Earth response, pertain to distinctly different parts of the Earth system. The excitation continuum measures mass motion on and within the Earth, due to climate and other processes, while the resonant response is a measure of the shape and deformability of the Earth. In this paper, we focus on the excitation process, taking the response to be a property of the Earth which changes slowly with respect to human time scales. That is, we assume that the Chandler frequency and damping are constant over the period of the available data (about 100 years). Our focus is on understanding the excitation continuum, and in this sense, the resonance is a nuisance to be removed from the data. Devising a numerical scheme for removing this resonance is a problem that was solved by Harold Jeffreys (1940), as discussed below.

The continuum model was not the concept of polar motion invoked at the time of Chandler's discovery in the 1890s. Instead, Chandler found it useful to simply describe the observed variations, without making assumptions about the physics. After all, physicists had been a problem in the past. Euler, Kelvin, and others had predicted a period near 300 days, and the search for this specific period of motion undoubtedly delayed the discovery by a century or more. Thus, there was some value at the time in promoting a conceptual model which was free of physics, one which succinctly summarized the data as a sum of discrete components, including the prominent Chandler and annual terms, and later others to describe long period motion. Today, it is recognized that while 'component' models such as introduced by Chandler, are effective in describing polar motion, they may not be helpful in understanding the physical processes which force it.

While component and continuum models are not mutually exclusive, the adoption of a component model may lead to differences relative to continuum models. As an example, consider the long-standing search for the excitation of the Chandler Wobble. This quest predates modern space geodetic data, and while it appears to be a well defined problem, it has commonly lacked specific definition in terms of the frequency band of polar motion to be explained. It is clear that a component model underlies the search for the Chandler Wobble excitation, since the Chandler Wobble is considered to be a distinct variation. When it is understood that a component model is the underlying concept, it is clear why such searches have often been carried out independently of studies of the annual wobble. This is true, for example, of many investigations related to earthquake excitation of the Chandler wobble.

How would a continuum model lead to different lines of study? Certainly, the working hypothesis would be different in the case of a continuum model. As proposed by Jeffreys (1940) the simplest hypothesis is that the Chandler Wobble excitation source is similar to that of the annual wobble, given that their periods (12 and 14 months) are nearly the same. Because there is no question that the annual wobble is forced by climate processes, the Chandler Wobble is therefore likely to be caused by the same processes. Following this line of reasoning an additional step, the degree to which one understands the annual wobble is then a reasonable measure of how well climate-related processes are understood, and can be used to judge how well one should expect the nearby Chandler wobble to be accounted for. An added strength of the continuum model is that it requires specification of a precise frequency range of study, and indeed leads one to seek an explanation for polar motion in any frequency band where the signal to noise 
level is reasonably good. Thus, the frequency band near the Chandler frequency is of interest, but also (with modern data) frequencies over a much broader band.

Another example shows how component and continuum concepts lead to divergent interpretations. It is well-known that power spectral analysis of long polar motion time series, such as the ILS series, reveals a split spectral peak near the Chandler frequency. Closely related to this is the finding that the Chandler peak has a variable frequency over time, if one analyzes short segments of the series. These properties of the data are not in dispute, but their interpretation has been. How do component and continuum models differ in this interpretation? From a component model point of view, a split peak might imply that the Earth has two distinct resonant frequencies. That is, a component model leads one to propose more components to describe the data. The apparent change in Chandler frequency over time might be understood as a change in the relevant physical properties of the Earth. Either the existence of multiple resonances, or a changing resonant frequency requires a new physical model for the Earth, one which would not follow from the customary development that begins with Euler's rigid body equations. On the other hand, the continuum model explains both a split power spectral peak and an apparent Chandler frequency change over time as consequences of variability in the excitation process. This interpretation is simpler, and requires no new physics.

\section{Applying Component and Continuum Models}

The application of a component model is relatively simple, requiring only a standard method, such as least-squares, to fit the specified components to the polar motion data. This makes it attractive to use, and explains its widespread appearance in the literature. The continuum model is more difficult to apply in a quantitative way. One must develop a way to separate the two elements in the model, the excitation process and the resonant response which filters it. That is, one needs a method of estimating the resonant response properties, and then removing their effect from the polar motion data in order to obtain the excitation time series. In practice this is done using Euler's equations as the model of the response to derive a digital filter, in combination with estimated Chandler wobble parameters (resonant frequency $F$ and quality factor $Q$ ). An early application of this general approach was by Jeffreys (1916) who investigated climate processes that contributed to the forcing of the annual motion. He showed that polar motion is a highly sensitive measure of variability in the oceans, biosphere, atmosphere, and hydrosphere, and he developed and applied the theory of inverted barometer response of the oceans to atmospheric pressure loading, an approach which remains the standard treatment of the problem.

While Jeffreys' 1916 paper examined a single frequency component of polar motion, an investigation of the broad-band excitation requires a digital filter whose behavior is inverse to that of the Earth, removing amplitude and phase variations due to the resonant response of the Earth. The development of a digital polar motion filter for this purpose was again the work of Jeffreys (1940). From today's perspective, the work was decades ahead of its time; digital filter design was not pursued on a wide scale until the development of digital computers in the 1950 s and 1960 s. 
Wilson and Haubrich (1976), Wilson (1985), and Wilson and Chen (1996) used modern digital filter design methods to refine Jeffreys original result, and these filters have been employed in a variety of studies to compute what is termed the astronomically or geodetically inferred excitation time series from the polar motion data. The inferred excitation can be compared directly with global integrals of mass redistribution and motion of air, water and other elements in the Earth system using formulations such as those given by Munk and MacDonald (1960) that follow from the Liouville equations. The coefficients of the inverse polar motion filter are determined once two parameters, the resonant Chandler frequency $F$ and the dissipation-related term, the quality factor $Q$, are set. We adopt the usual complex-valued notation for the polar motion at time $\mathrm{t}, M_{t}=\left(m_{1}+\imath m_{2}\right)$ where the $\mathrm{x}$ component is $m_{1}$ and the negative $\mathrm{y}$ component is $m_{2}$. The corresponding excitation time series $X_{t}=\left(\chi_{1}+\imath \chi_{2}\right)$ is obtained, in the form presented by Wilson (1985) as:

$$
X_{t}=\left\{\imath \exp (-\imath \pi F T) /[F T(1+\imath / 2 Q]\}\left\{M_{t+T / 2}-\exp [\imath(\pi F T(1+\imath / 2 Q))] M_{t-T / 2}\right\}\right.
$$

Commonly adopted values are $F=0.843$ cycles per year and $Q=175$ (Wilson and Vicente, 1997).

Jeffreys' (1940) motivation in developing the early version of equation (1) was to estimate the Chandler frequency and dissipation, and for this purpose, only the statistical properties of $X_{t}$ are relevant, and these are independent of phase and scaling modifications introduced by later authors. Jeffreys' estimators for Chandler frequency and damping were based on maximum likelihood principles, with the excitation assumed to be Gaussian white noise. Under this assumption, maximum likelihood estimates are values for which $X_{t}$ has minimum variance. Later work (Jeffreys, 1968) used various steps to improve the applicability of the Gaussian white noise assumption, as explained by Wilson and Vicente (1990).

There was little reason for Jeffreys to examine $X_{t}$ in detail given the poor quality of the ILS data available in 1940 and earlier. In these early data, the high noise level dominates $X_{t}$, except near Chandler and annual frequencies where the signal level is above the noise. In a later study, Munk and Hassan (1961) made use of newly available Fourier spectral analysis to focus their attention near the Chandler frequency where the signal to noise level is good. However, they analyzed $M_{t}$, rather than $X_{t}$, and their estimate of correlation suffered as a result, as noted by Wilson and Haubrich (1976). As a result, they concluded that there was no evidence of correlation between globally integrated barometric pressure data and observed polar motion, leaving the source of excitation for the Chandler wobble an open question. Their null finding inspired many subsequent students to abandon the hypothesis of climate-related forcing in search of other mechanisms, such as earthquakes. This new direction, by implication, abandoned the continuum conceptual model underlying the study of Munk and Hassan.

\section{Excitation Time Series}

Current practice is to adopt fixed values of $F$ and $Q$, compute $X_{t}$ via equation (1), and compare it with globally integrated air pressure, wind, and other time 
series to determine their relative contributions. The fact that $F$ and $Q$ are not known precisely does not affect the validity of the procedure. In fact, the choice of $F$ and $Q$, within reasonable limits, has very little influence on the conclusions because the inferred excitation changes only in a very narrow band near $F$.

Current studies take advantage of greatly improved understanding and global models of climate related sources of air and water mass redistribution and motion. Mature atmospheric general circulation models are available from a number of sources. Ocean general circulation models are available, though remain relatively immature compared with atmospheric models. Least welldeveloped are models of the hydrologic cycle, which are required to keep track of water balance over land. There are few fully coupled climate models which track water in all its forms in the atmosphere, oceans, and on land.

Given the high quality of polar motion data now available, the state of climate observation and modeling is the current limit to our understanding of polar motion. Discrepancies between the inferred polar motion excitation and predictions from atmospheric, ocean, and climate models are almost certainly due to shortcomings in one or more of the models, although it is not always possible to determine the source of the problem. This suggests that polar motion, because it is so precisely observed, has considerable value as a global measure of the accuracy and stability of climate models. This remains one of the unifying themes of modern studies of polar motion and other space geodetic time series.

Figures 1 and 2 are useful summaries of current understanding concerning the excitation. They show the time series for the two orthogonal excitation components ( 0 and 90 degrees East longitude). Various estimates of climate-related contributions to the excitation series are shown for 1993-1998, a period when excellent space geodetic data are available. We use equation (1) to determine the inferred excitation from the space geodetic time series Space97, which is a combined GPS,VLBI, and Laser Ranging solution given at daily intervals. Estimates of atmosphere, ocean and hydrologic contributions are derived from models and observations, as discussed below, and are intended to be representative of the current understanding of their relative contributions.

The two upper curves in Figures 1 and 2 show the contribution of the atmosphere, from the NCEP (National Center for Environmental Prediction) climate reanalysis model. Air mass effects are derived using Jeffreys' Inverted Barometer treatment to represent the load over the oceans. The wind effect, though showing lower variance, is still significant compared to air mass redistribution, especially at higher frequencies, well above 1 cycle per year.

The effects of continental water storage, shown in the third curve, are also estimated from the NCEP model. The time series are clearly different in appearance, showing only seasonal variations, repeating every year. Although the estimates show no shorter or interannual changes, hydrologic effects are actually expected to be broad band. Apparently the NCEP hydrologic scheme relies mostly on climatology, rather than numerical calculations to provide its estimates. The actual hydrologic excitation is expected to differ greatly. A seasonal cycle should be prominent, but it is anticipated that long-period variations should be pronounced, because water is able to accumulate in various locations (oceans, ice, lakes, ground water). This ability distinguishes it from atmospheric mass, which cannot easily accumulate in this way. The expectation is that water 


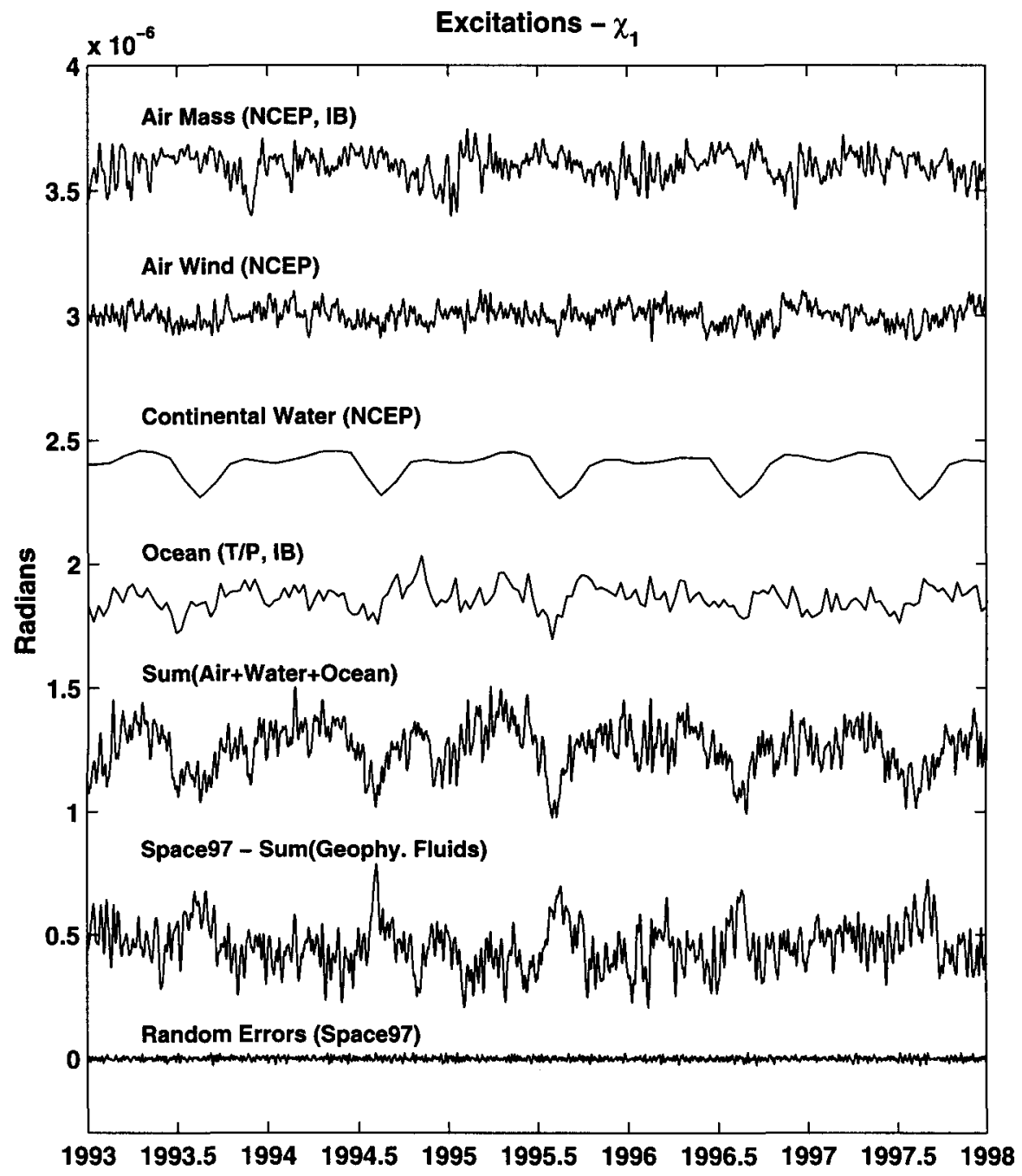

Figure 1. Excitation time series for the component along the Greenwich Meridian. The horizontal scale is calendar years and the vertical scale is radians. The mean value of each series is adjusted to arrange the series on the page, so as not to overlap. Each series is explained in the text. 


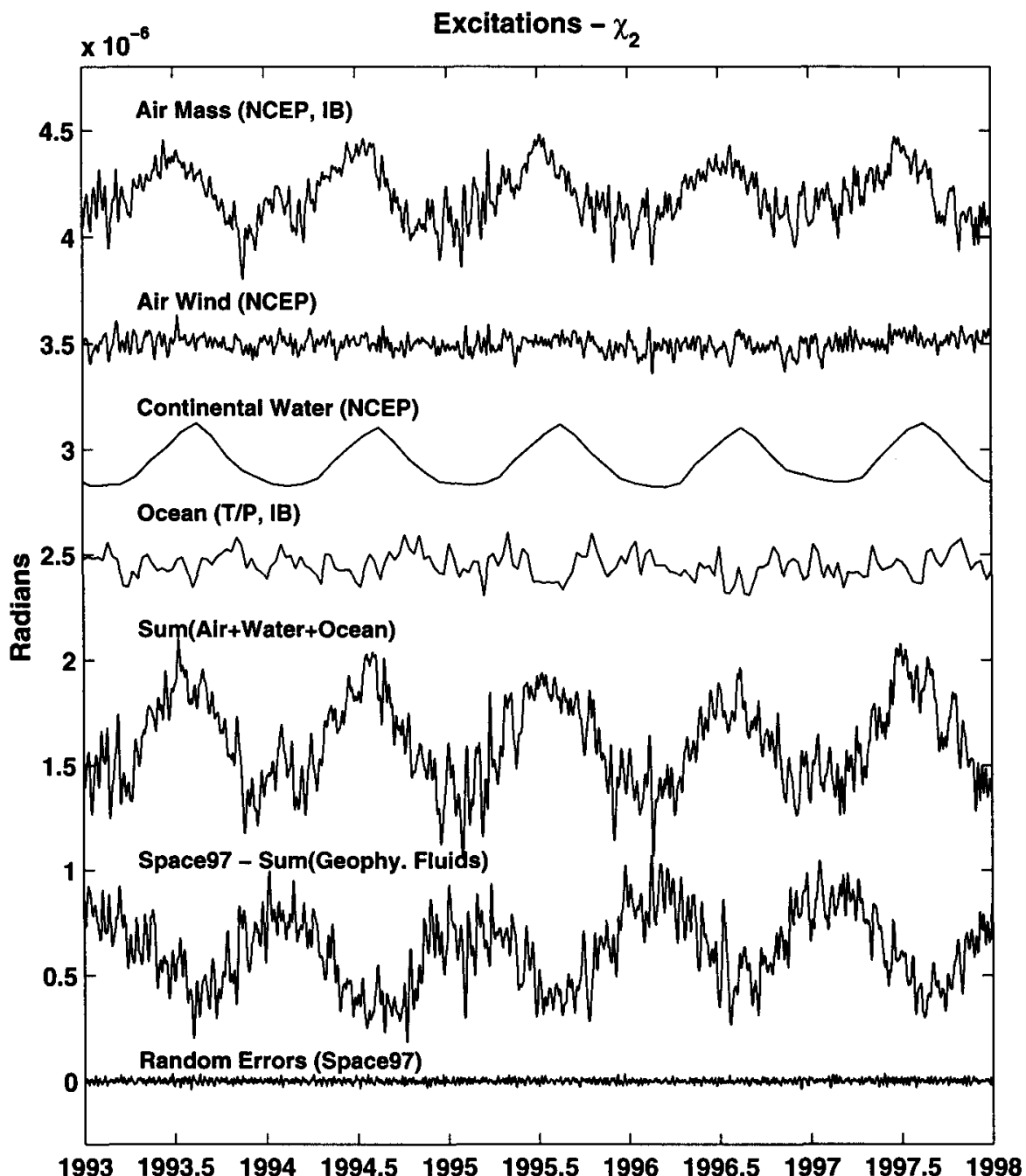

Figure 2. This figure shows the component along 90 degrees East Longitude. The horizontal scale is calendar years and the vertical scale is radians. The mean value of each series is adjusted to arrange the series on the page, so as not to overlap. Each series is explained in the text. 
storage effects will become more important, as atmospheric effects diminish in importance at longer periods. Of course, observed polar motion does show long period changes, on decadal and other time scales, and this motion is apparently well above the noise level in the data. This suggests that long period polar motion may be able to provide a useful measure of longer term climate changes, especially those related to movement of water mass among the various reservoirs in the Earth system.

The estimate of ocean effects shown here is from TOPEX satellite radar altimetry measurements of sea surface height after corrections for thermal expansion effects based on climatology. The details of this work are given by Chen et al., (1998), and the estimate omits any possible ocean current effects. Other estimates of ocean effects from numerical models, including results presented at this symposium, show similar magnitudes of effects, and are able to provide ocean current estimates, as well.

The residual excitation, which is the geodetically inferred time series minus the sum of the geophysical fluid estimates, is shown at the bottom, along with a time series generated by a random number generator to simulate the appearance of the noise level in the geodetically inferred excitation. The size of the residual is significant - comparable to the sum of geophysical fluids, and well above the noise level. The low variance of the noise time series makes it clear that virtually every variation in the residual series is significant.

\section{Conclusions}

Harold Jeffreys provided fundamental contributions to aid the transition from component to continuum conceptual models. He developed the most important analysis tool - the digital polar motion filter - which underlies today's studies investigating the broad-band continuum of the excitation function. The data from Jeffreys' time were not of sufficient quality to permit such studies. Modern space geodetic polar motion data are so good, that it is now climate data and models which limit our understanding of the continuum of polar motion excitation. This is evident in the size of the residual in Figures 1 and 2 which is comparable to the geophysical fluid predictions. The residual shows broad band variations, including strong annual terms, which clearly indicate the state of ignorance regarding climate variability in a global sense. It is now clear that all three excitation sources, atmosphere, oceans, and hydrology, provide comparable contributions to the excitation continuum. The air of mystery concerning the Chandler wobble excitation that Munk and Hassan created in their null finding in 1961 is now seen to be not only a consequence of their errors in spectral analysis, but also of the fact that the atmospheric mass contribution, the only one they examined, represents only about a third of the total excitation.

Improvements over the present situation are likely to come from two parallel lines of effort. One is from the climate model community, seeking better ocean, atmosphere, hydrosphere, cryosphere, and fully coupled models as part of practical questions related to understanding global climate. The other source of progress is expected to come from development of new space geodetic observations, especially from satellite-based gravity missions, which will achieve high spatial resolution measures of mass variation over the planet, leading to improved 
constraints on global water mass balance. Space-based gravity measurements, plus observations of geocenter, length of day, and zonal gravity changes from satellite laser tracking will greatly increase knowledge of climate-related mass redistribution and motion on our planet. Thus, modern geodesy has become a rich interdisciplinary field, closely connected with understanding of the global climate, a result firmly demonstrated in Jeffreys' 1916 paper.

\section{References}

Chen, J.L., C.R. Wilson, D.P. Chambers, R.S. Nerem, and B.D. Tapley, 1998. Seasonal global water mass balance and mean sea level variations, Geophys. Res. Lett., 15 (19), 3555-3558.

Jeffreys, H., 1916, Causes Contributory to the Annual Variation of Latitude, Monthly Notices Royal Astronomical Society, 76, 499-525.

Jeffreys, H., 1940. The Variation of Latitude. Monthly Notices Royal Astronomical Society, 100, 139-155.

Jeffreys, H., 1968. The Variation of Latitude, Monthly Notices Royal Astronomical Society, 141, 255-268.

Munk, W. and MacDonald, G., 1960. The Rotation of the Earth, a Geophysical Discussion, Cambridge University Press, 323 pp.

Munk, W., and Hassan, E., 1961. Atmospheric Excitation of the Earth's Wobble, Geophysical Journal Royal Astronomical Society, 4, 339.

Wilson, C. and Haubrich, R., 1976. Meteorological Excitation of the Earth's Wobble, Geophysical Journal Royal Astronomical Society, 46, 707.

Wilson, C., 1985, Discrete Polar Motion Equations, Geophysical Journal Royal Astronomical Society, 80, 551-554.

Wilson, C. and Vicente, R., 1990. Maximum Likelihood Estimates of Polar Motion Parameters, American Geophysical Union Geophysical Monograph 59, Variations in Earth Rotation, D. McCarthy and W. Carter Editors.

Wilson, C., and Chen, J., 1996. Discrete Polar Motion Equations for High Frequencies. Journal of Geodesy. v. 70, no. 9.

Vicente, R., and Wilson, C., 1997, On the Variability of Earth Rotation Parameters, Journal of Geophysical Research, 102, B9, 20439-20445. 


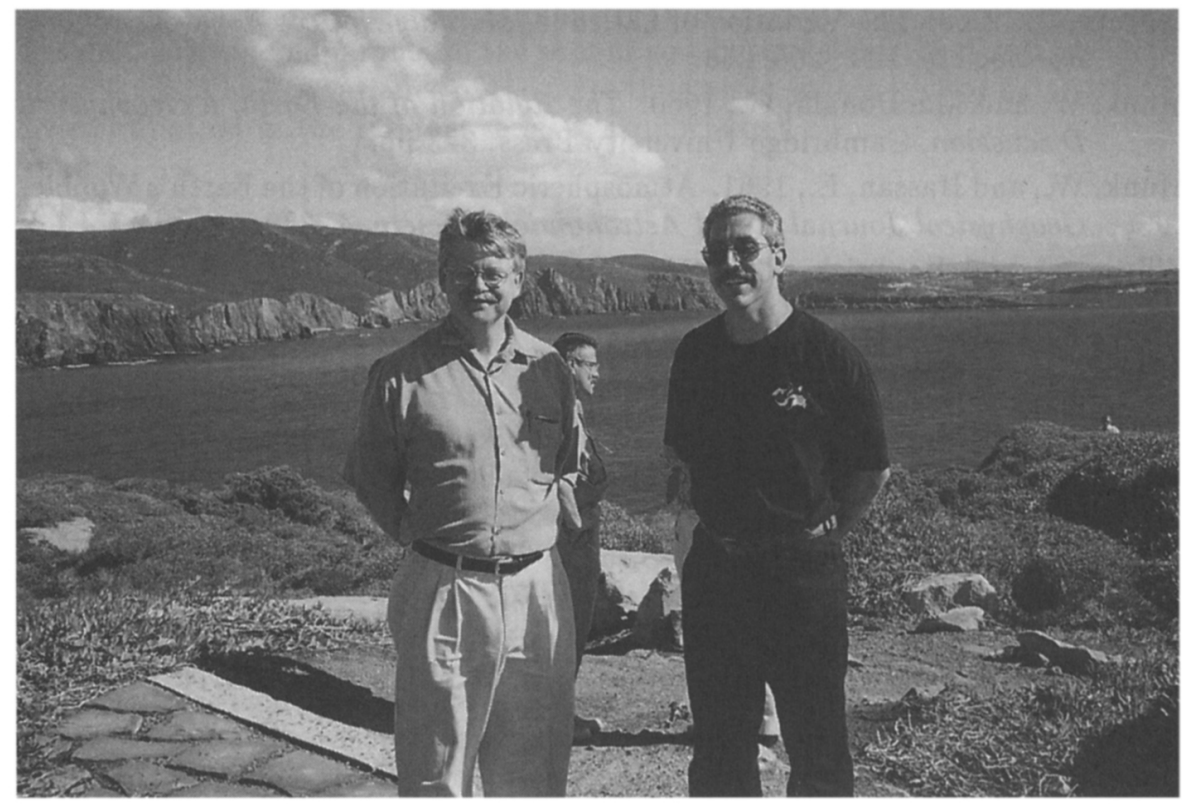

Steve Dick and Steve Dickman 